\title{
Spatial Heterogeneity of the Incidence of Powdery Mildew on Hop Cones
}

\author{
David H. Gent, United States Department of Agriculture-Agricultural Research Service (USDA-ARS), Forage Seed \\ and Cereal Research Unit, and Walter F. Mahaffee, USDA-ARS, Horticultural Crops Research Laboratory, Oregon \\ State University, Department of Botany and Plant Pathology, Corvallis 97331; and William W. Turechek, USDA- \\ ARS, Fruit Laboratory, Beltsville, MD 20705-2350
}

\begin{abstract}
Gent, D. H., Mahaffee, W. F., and Turechek, W. W. 2006. Spatial heterogeneity of the incidence of powdery mildew on hop cones. Plant Dis. 90:1433-1440.

The spatial heterogeneity of the incidence of hop cones with powdery mildew (Podosphaera macularis) was characterized from transect surveys of 41 commercial hop yards in Oregon and Washington from 2000 to 2005 . The proportion of sampled cones with powdery mildew $(\hat{p})$ was recorded for each of 221 transects, where $N=60$ sampling units of $n=25$ cones assessed in each transect according to a cluster sampling strategy. Disease incidence ranged from 0 to 0.92 among all yards and dates. The binomial and beta-binomial frequency distributions were fit to the $N$ sampling units in a transect using maximum likelihood. The estimation procedure converged for $74 \%$ of the data sets where $\hat{p}>0$, and a log-likelihood ratio test indicated that the beta-binomial distribution provided a better fit to the data than the binomial distribution for $46 \%$ of the data sets, indicating an aggregated pattern of disease. Similarly, the $C(\alpha)$ test indicated that $54 \%$ could be described by the beta-binomial distribution. The heterogeneity parameter of the beta-binomial distribution, $\hat{\theta}$, a measure of variation among sampling units, ranged from 0.01 to 0.20 , with a mean of 0.037 and a median of 0.015 . Estimates of the index of dispersion ranged from 0.79 to 7.78 , with a mean of 1.81 and a median of 1.37 , and were significantly greater than 1 for $54 \%$ of the data sets. The binary power law provided an excellent fit to the data, with slope and intercept parameters significantly greater than 1 , which indicated that heterogeneity varied systematically with the incidence of infected cones. A covariance analysis indicated that the geographic location (region) of the yards and the type of hop cultivar had little effect on heterogeneity; however, the year of sampling significantly influenced the intercept and slope parameters of the binary power law. Significant spatial autocorrelation was detected in only $11 \%$ of the data sets, with estimates of first-order autocorrelation, $\hat{r}_{1}$, ranging from -0.30 to 0.70 , with a mean of 0.06 and a median of 0.04 ; however, correlation was detected in only 20 and $16 \%$ of the data sets by median and ordinary runs analysis, respectively. Together, these analyses suggest that the incidence of powdery mildew on cones was slightly aggregated among plants, but patterns of aggregation larger than the sampling unit were rare (20\% or less of data sets). Knowledge of the heterogeneity of diseased cones was used to construct fixed sampling curves to precisely estimate the incidence of powdery mildew on cones at varying disease intensities. Use of the sampling curves developed in this research should help to improve sampling methods for disease assessment and management decisions.
\end{abstract}

Additional keywords: Humulus lupulus

Hop (Humulus lupulus) is an economically important crop in the Pacific Northwest of the United States, which produces nearly the entire U.S. supply and greater

Corresponding author: D. H. Gent

E-mail: gentd@ onid.orst.edu

The use of trade, firm, or corporation names in this publication is for the information and convenience of the reader. Such use does not constitute an official endorsement or approval by the United States Department of Agriculture or the Agricultural Research Service of any product or service to the exclusion of others that may be suitable.

Accepted for publication 27 June 2006.

DOI: $10.1094 / P D-90-1433$

This article is in the public domain and not copyrightable. It may be freely reprinted with customary crediting of the source. The American Phytopathological Society, 2006. than $30 \%$ of the world supply of hop (9). The cones of the female plant are used almost exclusively for imparting flavor and aroma to beer. Hop production in the $\mathrm{Pa}$ cific Northwest generates over $\$ 100$ million in farm gate value annually (1).

Powdery mildew, caused by Podosphaera macularis Braun \& Takamatus, is one of the most important diseases of hop worldwide (20). The disease occurs on leaves and petioles, but economic losses are associated primarily with infection of inflorescences (burrs) and developing cones. Although infections of leaves are rarely economically damaging, they provide inoculum to initiate infections of burrs and cones and must be managed to minimize cone infection $(24,32)$. Severe burr or cone infection may cause complete loss of marketable yield through abortion of burrs and young cones, quality losses, and reductions in $\alpha$-acid (which imparts bitterness to beer).

Successful control of hop powdery mildew requires an integration of diverse management tactics (24). Cultural practices such as thorough chemical or mechanical pruning, removal of excessive basal foliage, and sanitation of infested crop debris can reduce disease but, alone, are insufficient to control the disease $(18,20,32)$. Cultivars vary widely in their resistance or tolerance to powdery mildew $(20,24)$, but complete resistance is not found among commercially acceptable cultivars sought by most brewers. Susceptible cultivars such as Willamette constitute a large portion of the aroma hops used in some premium beers. The flavor and aroma profile are specific and dictate the production of certain cultivars. Consequently, growers rely heavily on intensive fungicide applications for disease management. Because hop produces prodigious growth of susceptible tissue, fungicides are applied throughout the entire season to protect newly emerged leaves, although the risk of disease varies with temperature and rainfall (19).

Although several strategies for managing hop powdery mildew have been developed, the basic elements of the spatial and temporal patterns of the disease on cones are lacking in the literature. This is surprising given that economic losses from hop powdery mildew are attributed largely to cone infections and associated expenses for disease management. This lack of understanding has limited our ability to develop innovative management tactics and testable hypotheses that link patterns of disease development to biological processes. Description and quantification of spatial patterns often are considered prerequisites to understanding basic elements of disease epidemiology, gaining insights into inoculum sources and dispersal mechanisms of the pathogen, and, ultimately, developing sound management tactics $(2,23)$. Knowledge of spatial patterns also allows for the rational design of sampling methods $(4,8,12,13,28,30)$, construction of crop loss models $(3,5)$, and controlling the effects of aggregation in the analysis of designed experiments (17).

Turechek and Mahaffee (31) characterized the spatial patterns of hop powdery mildew in commercial hop yards, found that the pattern of disease on leaves was 
largely random, and developed a sampling method for rapidly assessing disease incidence. However, the spatial pattern of disease on cones may not necessarily be the same as on leaves. Hop leaves display ontogenic resistance (32), but such resistance is not known to occur in cones. Additionally, cones are susceptible to infection for a much longer time than leaves $(24,25)$. A sampling plan for estimating the incidence of powdery mildew on hop cones has not been developed, but may be useful for assessing the efficacy of control measures and for development of management-action thresholds for the foliar phase of the disease. The purpose of this research was to (i) characterize the spatial pattern of hop powdery mildew on cones; (ii) determine whether patterns of disease incidence on cones are associated with geographic region, hop cultivar, or season; and (iii) use the knowledge of disease heterogeneity-variability among sampling units indicative of aggregation - to develop efficient sampling curves.

\section{MATERIALS AND METHODS}

Field sites and data collection. The incidence of hop powdery mildew on cones was assessed for 221 transects (data sets) sampled in 41 commercial hop yards in the primary hop-growing regions of Oregon (Willamette Valley) and Washington (Yakima Valley and surrounding areas) from 2000 to 2005. All Oregon yards included in this study were located in Marion County. Yards in Washington were located in three separate regions: (i) the Yakima Indian Reservation, ii) near the town of Moxee City (Moxee), and (iii) in the eastern extent of the Yakima Valley (Lower Valley), described as the Prosser and Mabton regions by Turechek and Mahaffee (31). The severity of hop powdery mildew varies among these regions partly because of regional climate differences and grower cultural practices $(1,31)$, and generally is greatest in and around the Yakima Indian Reservation, followed by Moxee, the Lower Valley, and Oregon.

Cultural practices varied among yards in Oregon and Washington. Most yards in Oregon were planted with a 2.1-by-2.1-m spacing between plants and rows, respectively, whereas most yards in Washington were planted with a 1-by-4.25-m spacing. Yards in Oregon were irrigated mostly by sprinkler or, less commonly, surface drip irrigation, whereas yards in Washington were irrigated mostly by surface drip or, less commonly, furrow irrigation. Yards surveyed in Oregon were planted exclusively with the aroma cvs. Glacier (3 yards), Horizon (2 yards), Liberty (2 yards), Perle (4 yards), Sterling (1 yard), and Willamette (26 yards) that are moderately to highly susceptible to hop powdery mildew. Yards surveyed in Washington were planted primarily with the bittering cvs. Chelan (1 yard), Columbus, Toma- hawk, or Zeus (genetically indistinguishable and collectively referred to as CTZ) (42 yards), Galena (3 yards), or Warrior (1 yard) that are highly susceptible to hop powdery mildew (31). Ten yards of the aroma cvs. Horizon (one yard) and Willamette (nine yards) also were surveyed in Washington.

Disease incidence was assessed using a cluster sampling design (7). In 2000, yards were stratified into $H$ strata, where $H=$ number of rows in a yard/20 (rounded up to the nearest integer), and one transect (row) in each of the strata was selected arbitrarily for sampling. Because of the large amount of time and labor necessary to harvest and assess cones for powdery mildew, only one transect was assessed from the first 40 rows on the north or east side (30) of each yard from 2001 to 2005. Cones were sampled from the first $60(N)$ plants along the transect by collecting cones from lateral branches at heights of approximately $2.7,3.7$, and $5.5 \mathrm{~m}$ from the ground. The cones from each sampling unit (plant) were bulked before selecting $25(n)$ cones arbitrarily from each plant for a total of 1,500 cones per transect. Each cone was evaluated for signs of mycelia of $P$. macularis with the aid of a dissecting microscope, when needed, within $48 \mathrm{~h}$ of collection. Mean incidence of diseased cones $(\hat{p})$ was calculated as:

$$
\hat{p}=\sum X_{i} / \sum n_{i}
$$

where $X_{i}$ is the number of diseased cones and $n_{i}$ is the number of leaves sampled in the $i$ th sampling unit. Occasionally, less than 25 cones inadvertently were sampled from each plant. Therefore, the mean number of cones per transect was calculated as:

$$
\bar{n}=\sum n_{i} / N
$$

In Oregon, cones were assessed from 56 transects among 11 yards in 2000 . As stated earlier, only one transect was assessed from each transect from 2001 to 2005 . Therefore, the number of transects sampled from 2001 to 2005 also represents the number of yards sampled. In all, 14 transects were sampled in each of 2001 and 2002, 4 transects in each of 2003 and 2004, and 8 transects in 2005. In Washington, cones were assessed from 70 transects among 26 yards in 2000 . Cones were assessed from 27, 7, 5, 4, and 8 transects in 2001, 2002, 2003, 2004, and 2005, respectively.

Point-pattern beta analyses. Distributional analyses. The beta-binomial and binomial distributions were fit to the incidence of diseased cones using the computer program BBD (10). A good fit to the binomial distribution is an indication of a random pattern of disease, whereas a good fit to the beta-binomial distribution is an indication of an aggregated pattern $(11,13)$. A log-likelihood ratio test statistic was calculated to determine whether the data was a better fit to the beta-binomial distribution or the binomial distribution. The
$\mathrm{C}(\alpha)$ test was used to test whether aggregation in the distribution of infected cones could be described adequately by the betabinomial distribution $(11,22)$. It was not possible to calculate expected frequencies or perform $\chi^{2}$ goodness-of-fit tests for the beta-binomial or binomial distributions because $n$ was not constant among plants.

The degree of aggregation was quantified by the parameter $\theta$ of the betabinomial distribution, which provides a measure of variation in disease incidence per sampling unit generally referred to as heterogeneity (29), and the index of dispersion, $D$ (11). The index of dispersion is the ratio of the observed variance of the incidence of disease among sampling units to the expected variance under a binomial distribution, and is directly analogous to the variance-to-mean ratio of count data with a Poisson (random) distribution (11). The variance of the proportion of diseased cones, $v_{\mathrm{obs}}$, was calculated as:

$$
v_{\mathrm{obs}}=\left[\sum n_{i}^{2}\left(p_{i}-\hat{p}\right)^{2}\right] /\left[n^{-2}(N-1)\right]
$$

where $p_{i}=x_{i} / n$ and $\hat{p}$ are as defined above. The variance for a binomial distribution $\left(v_{\text {bin }}\right)$ was calculated as:

$$
v_{\text {bin }}=\hat{p}(1-\hat{p}) / \bar{n}
$$

When $D=1$ or $\theta=0$ the incidence of disease is random. Aggregation is indicated when $D>1$ or $\theta>0$, with the degree of aggregation directly proportional to the magnitude of the statistic. The index of dispersion has a $\chi^{2}$ distribution and can be used to test the null hypothesis of a random distribution of disease incidence with $N-1$ degrees of freedom (11).

Binary power law analyses. The observed and binominal variances were fit to the binary form of Taylor's power law $(5,26,27)$ given by:

$$
\ln \left(v_{\text {obs }}\right)=\ln \left(A_{p}\right)+b \ln [\hat{p}(1-\hat{p}) / \bar{n}]
$$

where $\ln \left(A_{p}\right)$ and $b$ are the intercept and slope parameters of a straight line, respectively. When $A_{p}=1$ and $b=1$, equation 5 indicates a random pattern of diseased cones that can be represented by the binomial distribution. When $A_{p}>1$ and $b=1$, disease incidence has an aggregated pattern, but aggregation is not dependent on $p$. Values of $b>1$ indicate that aggregation is systematically related to $p$. When $b>1$, the beta-binomial parameter $\theta$ can be expressed in terms of $A_{p}$ and $b$ of the binary power law according to the formula:

$\theta=[a-f(p) / n] /[f(p)-a]$

where $f(p)=[p(1-p)]^{1-b}$ and $a=A_{p} n^{-b}$. Equation 6 represents a curve with maximum value at $p=0.5$ when $b>1$ and a horizontal line when $b=1$. Ordinary least squares regression was used to estimate the intercept and slope parameters using SAS (version 9.1, PROC REG; SAS Institute, Cary, NC).

A covariance analysis was conducted to determine the effect of hop cultivar type, 
geographic region of the yards, and the year of sampling on the slope or intercept parameters of the binary power law, as described previously (29). Cultivars were classified as aroma (Glacier, Horizon, Liberty, Perle, or Willamette) or bittering (Chelan, CTZ, Galena, and Warrior). Yards were classified into regions based on their location (i.e., western Oregon, the Lower Valley, Moxee, and the Yakima Indian Reservation). The continuous variable $\ln [\hat{p}(1-\hat{p}) / \bar{n}]$ was included in the model first, and then each of the three factors was added individually as an intercept and then as an interaction term with the slope. The analyses were conducted on each year separately, and then a separate analysis was conducted to determine the effect of year on estimates of $\ln \left(A_{p}\right)$ and $b$. Covariance analyses were performed using the GENMOD procedure in SAS.

Correlation-based spatial analyses. Autocorrelation. First- and second-order spatial autocorrelation statistics were calculated to quantify the similarity (or dissimilarity) of disease incidence among cones of neighboring plants within a transect (29). Data were transformed using the logit transformation $\ln [y /(1-y)]$, where $y$ $=(x+0.5) /(n+1)$ is the Haldane transformation of $x$ and $x$ is the number of diseased cones in a sampling unit, prior to calculating spatial autocorrelation coefficients. Spatial autocorrelation analyses were not performed among plants in different transects because the location of these plants and transects were not georeferenced. Autocorrelation analyses were performed in S-Plus (version 6.2; Insightful Corp., Seattle, WA).

Runs analyses. Ordinary and median runs analyses were performed to characterize larger scale patterns of diseased cones among plants in a transect (16). For ordinary runs analysis, a plant was considered diseased if at least one diseased cone was observed in that sampling unit. For median runs analysis, the median incidence of disease was calculated for each data set. Plants were assigned a value of 1 or 0 if the incidence of disease was above or below the median, respectively. For both analyses, a run was defined as a succession of one or more plants with similar disease status (nondiseased or diseased; 16). The expected number of runs was calculated and used to produce a $Z$-statistic to test the null hypothesis that the number of runs was not different significantly $(P \leq 0.05)$ from the expected number of runs (i.e., an indication of a random distribution of disease among plants). Runs were calculated in Microsoft Excel (Microsoft Corp., Redmond, WA).

Sample size analyses. Sampling curves were developed to predict the number of sampling units necessary to estimate the incidence of diseased cones with varying levels of precision $(13,14)$. The number of sampling units $(N)$ needed to estimate $p$ with a coefficient of determination, $C$, of $0.2,0.3$, and 0.4 was calculated by:

$N=a p^{b-2}(1-p)^{b} / C^{2}$

where $C=$ standard error $[\hat{p}] / \hat{p}, a=A_{p} n^{-b}$, and $A_{p}$ and $b$ are parameters estimated from the binary power law (equation 5). For simplicity, it was assumed that $n=25$ and was constant among sampling units (14).

Disease was not observed in 20 of the 221 transects sampled. However, there is finite and nonzero probability that $p>0$ for these data sets (15). The probability of not observing disease in $N$ successive sampling units (when $n=25$ ) was quantified following the equation of Madden et al. (15):

$P \approx(1+n \theta)^{-N p / \theta}$

where $P=$ the probability of observing no diseased individuals in all of the sampling units, and $\theta$ was estimated from the mean $\hat{\theta}$ for Oregon (0.02) and Washington (0.05). This is the equation for the negative binomial distribution with mean $n p$ and aggregation parameter $p / \theta$, but approximates $P$ for the beta-binomial distribution when $p<0.10$ (13). When disease incidence is distributed according to a binomial distribution, the exact probability can be calculated by:

$P=(1-p)^{n N}$

Additionally, the upper limit of a $95 \%$ confidence interval for $p$ at different $N$ was calculated to determine the relationship among $p$ and $N$ when $\hat{p}$ is near zero, by:

$$
p_{u} \approx \frac{-\theta \log (P)}{N \log (1+n \theta)}
$$

The upper limit of the confidence interval for a theoretical binomial distribution was calculated by:

$p_{u}=1-P^{(1 / n N)}$

Lower limits of the confidence intervals were not calculated because $p<0$ is not possible. These calculations differ from the calculation of sample sizes according to equation 7 , in that equations 8 to 11 are special cases when the objective of sampling is to estimate disease incidence when $p$ is near 0 for a given $N, n$, and $\theta$. These sampling strategies are intended to fulfill different purposes depending on $p$ and, expectedly, may not estimate identical results for a given $p$.

\section{RESULTS}

Disease incidence. The incidence of hop powdery mildew on cones ranged from 0 to 0.92 among all yards, years, and regions, and displayed a right-skewed distribution (Table 1; Fig. 1A). Averaged over all years, disease incidence among regions was lowest in Oregon (mean of 0.02 , median of 0.01 , and range of 0.01 to 0.04), and highest near Moxee (mean of 0.18 , median of 0.05 , and range of 0.13 to 0.42 ). The incidence of disease generally increased in all production regions in Washington from 2000 to 2005. Among the 221 data sets (transects) assessed, $\hat{p} \leq$ 0.05 in greater than $72 \%$ of the datasets.

Point pattern analyses. Distributional analyses. The maximum likelihood estimation (MLE) procedure converged for 149 $(74 \%)$ of the data sets. The procedure converged in greater than $96 \%$ of the data sets where $\hat{p}>0.05$ (Table 2). The loglikelihood ratio test was significant for 92 $(46 \%)$ data sets where $\hat{p}>0$, indicating that the beta-binomial distribution provided a better fit to the data than the binomial (random) distribution for these data sets. The $C(\alpha)$ test indicated that the betabinomial distribution provided a better fit than the binomial distribution for 109 (54\%) of the data sets, which supported results of the log-likelihood ratio test. The frequency distribution of the heterogeneity parameter $\hat{\theta}$ was right skewed and ranged from 0.01 to 0.39 , with a mean of 0.037 and median of 0.015 among all yards surveyed, indicating a low degree of aggregation (Fig. 1B; Table 3). Aggregation was

Table 1. Mean incidence $(\hat{p})$ and standard error of the mean incidence of hop cones with powdery mildew from commercial hop yards in Oregon and Washington (Lower Valley, Moxee, and Yakima Indian Reservation [YIR]) ${ }^{\mathrm{a}}$

\begin{tabular}{|c|c|c|c|c|c|c|c|c|c|c|}
\hline \multirow[b]{2}{*}{ Year ${ }^{b}$} & \multicolumn{2}{|c|}{ Oregon } & \multicolumn{2}{|c|}{ Lower Valley } & \multicolumn{2}{|c|}{ Moxee } & \multicolumn{2}{|c|}{ YIR } & \multicolumn{2}{|c|}{ Total } \\
\hline & $\hat{\hat{p}}$ & $\mathbf{T}^{\mathbf{b}}$ & $\hat{\hat{p}}$ & $\mathbf{T}$ & $\hat{\hat{p}}$ & $\mathbf{T}$ & $\hat{\hat{p}}$ & $\mathbf{T}$ & $\hat{\hat{p}}$ & $\mathbf{T}$ \\
\hline 2000 & $0.01(0.003)$ & 56 & $0.02(0.004)$ & 35 & $0.14(0.187)$ & 15 & $0.10(0.026)$ & 20 & $0.02(0.003)$ & 126 \\
\hline 2001 & $0.01(0.004)$ & 14 & $0.07(0.027)$ & 9 & $0.13(0.070)$ & 7 & $0.10(0.042)$ & 11 & $0.07(0.018)$ & 41 \\
\hline 2002 & $0.02(0.011)$ & 14 & $0.38(0.165)$ & 2 & $0.32(\ldots)$ & 1 & $0.21(0.106)$ & 4 & $0.11(0.036)$ & 21 \\
\hline 2003-04 & $0.04(0.022)$ & 9 & $0.39(0.346)$ & 2 & $0.24(0.229)$ & 3 & $0.14(0.059)$ & 3 & $0.13(0.056)$ & 17 \\
\hline 2005 & $0.01(0.005)$ & 8 & $0.61(0.218)$ & 3 & $0.42(0.325)$ & 2 & $0.29(0.169)$ & 3 & $0.22(0.078)$ & 16 \\
\hline Total & $0.02(0.003)$ & 101 & $0.09(0.279)$ & 51 & $0.18(0.043)$ & 28 & $0.13(0.022)$ & 41 & $0.08(0.010)$ & 221 \\
\hline
\end{tabular}

a $\mathrm{T}=$ number of data sets (transects) assessed.

${ }^{\mathrm{b}}$ Field sites in each region were combined in 2003 and 2004 because of the small number of fields assessed in these years. 
slightly greater in Washington than Oregon, with a mean $\hat{\theta}$ of 0.049 (median of 0.02 ) for yards in Washington compared with 0.019 (median 0.01) for yards in Oregon. Consistent with equation $6, \hat{\theta}$ increased with increasing $\hat{p}$ except at the highest disease incidences (Fig. 2; Table 2 ). The frequency distribution of the index of dispersion, $D$, was right skewed and ranged from 0.79 to 7.78 , with a mean of 1.81 and median of 1.37 (Fig. 1C). In 108
(54\%) of the data sets, $D$ was greater than 1 according to a $\chi^{2}$ test.

Binary power law analyses. The binary power law provided an excellent fit to the data with coefficients of determination $\left(R^{2}\right)$ exceeding 0.95 for all years (Fig. 3; Table 4). The slope and intercept parameters were significantly greater than 1 and 0 , respectively, in all years $(P<0.05)$ based on a $t$ test. Estimates of the slope parameter were similar among years $(P>0.05)$
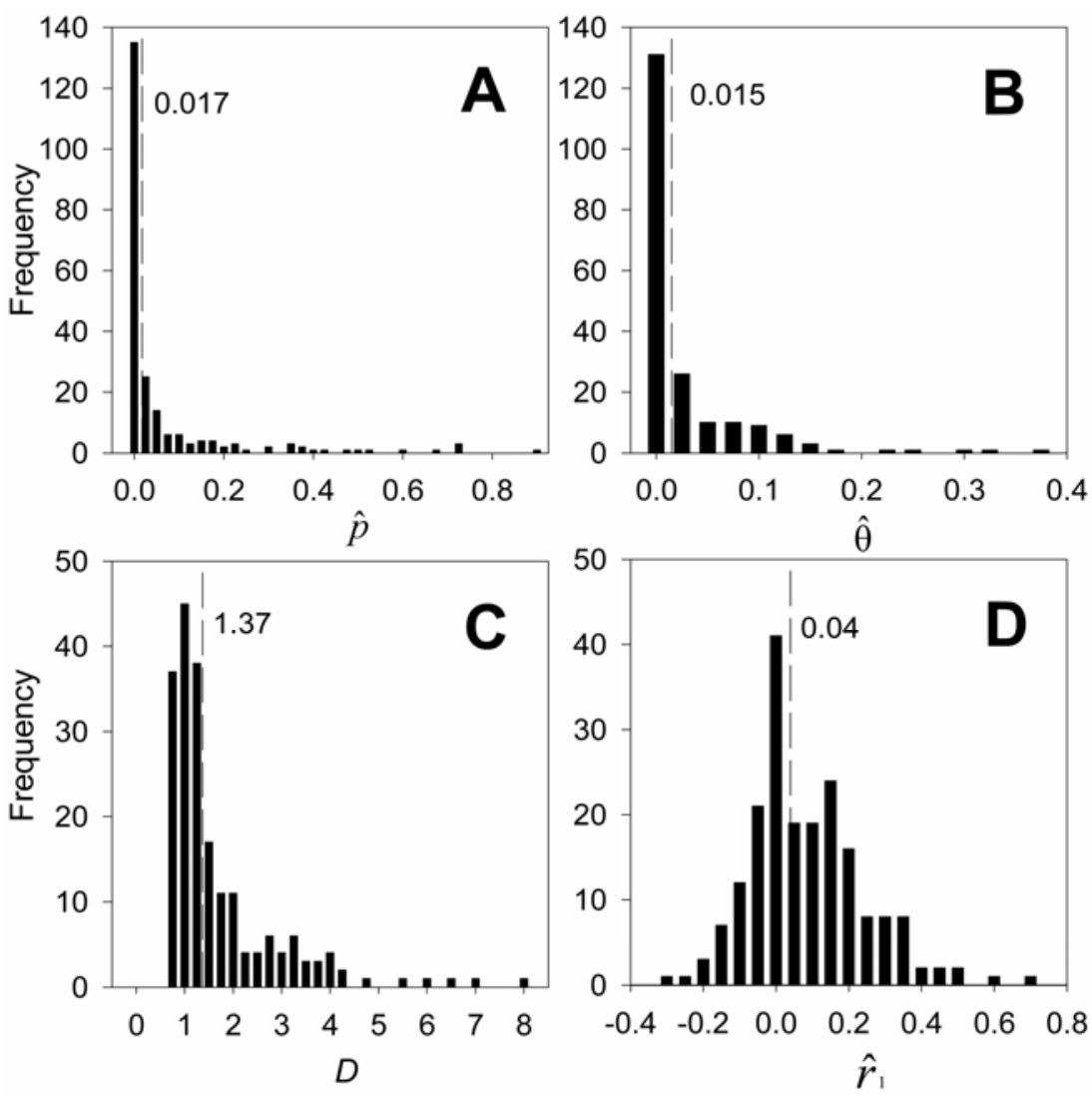

Fig. 1. Frequency distribution of the beta-binomial distribution parameters $\mathbf{A}, \hat{p}$ and $\mathbf{B}, \hat{\theta}, \mathbf{C}$, the index of dispersion $D$, and $\mathbf{D}$, the first-order autocorrelation statistic $\hat{r}_{1}$ for the incidence of hop cones with powdery mildew sampled from 221 transects in 41 commercial hop yards in Oregon and Washington from 2000 to 2005. The vertical dashed lines in each figure are the median value for the indicated statistic, with the numerical value given on the graph (11).

and ranged from 1.16 to 1.37 (Table 4). Estimates of the intercepts varied significantly among years $(P<0.05)$, ranging from 1.50 to 3.37 . This indicates that the incidence of diseased cones was slightly aggregated, and the degree of heterogeneity changed systematically with $p$ in all years.

The relationship between $\hat{\theta}$ and $\hat{p}$ predicted by equation 6 , where $\theta$ increases with $p$ until $p=0.5$, was generally true (Fig. 2); however, there was considerable variation in the individual values of $\hat{\theta}$ at any given $\hat{p}$, and the relationship was not exact, as found for other systems (29-31). The parameters of the binary power law were estimated by regression analysis and did not fit the observed data exactly (i.e., $R^{2}<1.0$ ). Because the binary power law compares variances on a logarithmic scale, small deviations in observed versus predicted values may result in large differences in values of $\hat{\theta}$ predicted by equation 6 (29).

The covariance analysis indicated that the slope and intercept parameter estimates did not vary with cultivar type or geographic region $(P>0.05$; Table 5). However, estimates of the intercept and slope parameters differed significantly with year $(P>0.0001)$, with a tendency to increase each year (Table 5). This indicates that heterogeneity of diseased cones increased with each successive year.

Correlation-based spatial analyses. Autocorrelation. Significant first-order spatial autocorrelation was detected in 21 $(11 \%)$ of the data sets and in only $1(0.5 \%)$ transect at lag $=2$. An association between $\hat{r}_{1}$ and $\hat{p}$ was not apparent (Table 2). The frequency distribution of the first-order spatial autocorrelation statistic, $\hat{r}_{1}$, was right skewed, with a range of -0.30 to 0.70 , a mean of 0.06 , and a median of 0.04 (Fig. 1D).

Runs analyses. Significant $(P<0.05)$ aggregation was detected in $40(20 \%)$ data sets by median runs analysis and $33(16 \%)$ data sets by ordinary runs analysis (Table

Table 2. Tests of aggregation and spatial pattern of the incidence of hop cones with powdery mildew sampled from commercial hop yards in Oregon and Washington in 2000 to 2005

\begin{tabular}{|c|c|c|c|c|c|c|c|c|c|c|}
\hline \multirow[b]{2}{*}{ Incidence $^{d}$} & \multirow[b]{2}{*}{$T^{\mathrm{e}}$} & \multirow[b]{2}{*}{ MLE $^{\mathrm{f}}$} & \multirow[b]{2}{*}{ LRS $^{\prime}$} & \multicolumn{2}{|c|}{ Variance tests $^{\mathbf{a}}$} & \multicolumn{3}{|c|}{ Median $^{\text {b }}$} & \multicolumn{2}{|c|}{ Runs analysis $^{\mathrm{c}}$} \\
\hline & & & & $C(\alpha)$ & $D$ & $\hat{\theta}$ & $D$ & $\hat{r}$ & Median & Ordinary \\
\hline 0 & 20 & & & & & & & & & \\
\hline $0.00-0.02$ & 99 & 54.5 & 19.2 & 28.3 & 28.3 & 0.01 & 1.14 & -0.017 & 17.2 & 17.2 \\
\hline $0.02-0.05$ & 41 & 87.8 & 51.2 & 65.9 & 65.9 & 0.02 & 1.45 & 0.12 & 24.4 & 14.6 \\
\hline $0.05-0.10$ & 20 & 90.0 & 65.0 & 75.0 & 70.0 & 0.02 & 1.58 & 0.049 & 10.0 & 5.0 \\
\hline $0.10-0.20$ & 17 & 100.0 & 94.1 & 94.1 & 94.1 & 0.06 & 2.36 & 0.069 & 29.4 & 5.9 \\
\hline $0.20-0.40$ & 13 & 100.0 & 92.3 & 92.3 & 92.3 & 0.10 & 3.40 & 0.001 & 7.7 & 23.1 \\
\hline $0.40-0.80$ & 10 & 100.0 & 100.0 & 100.0 & 100.0 & 0.18 & 4.68 & 0.171 & 0 & 40.0 \\
\hline $0.80-1.00$ & 1 & 100.0 & 100.0 & 100.0 & 100.0 & 0.13 & 3.85 & -0.056 & 0 & 0 \\
\hline
\end{tabular}

a Percentage of datasets in which the $C(\alpha)\left(Z\right.$-statistic) or $D\left(\chi^{2}\right)$ tests were significant $(P \leq 0.05)$.

${ }^{\mathrm{b}}$ Median estimated value of the beta-binomial distribution parameter $\hat{\theta}$, index of dispersion $D$, and first-order autocorrelation statistic.

${ }^{c}$ Percentage of datasets in which runs analysis indicated significant aggregation.

${ }^{\mathrm{d}}$ Incidence of hop powdery mildew on cones. Disease incidence class ends with the indicated value. Classes start with the next highest value above the listed value.

e Number of datasets (transects) in each disease incidence class.

${ }^{\mathrm{f}}$ Percentage of datasets in which the maximum likelihood estimation procedure converged.

g Percentage of datasets in which the likelihood ratio test statistic was significant $(P \leq 0.05)$. 
2). Among the disease incidence classes evaluated, there was no apparent pattern or association between aggregation and $\hat{p}$ as determined by visual inspection of scatter plots of $\hat{p}$ and the standard-normal $Z$ statistic (data not presented).

Sample size analyses. As expected, a larger sample size was required for low $C$ values (i.e., higher precision) and to estimate disease when $\hat{p}$ was low (Fig. 4A). At $\hat{p}=0.01$, approximately 132,60 , or 33 plants would need to be sampled to estimate disease incidence with $C=0.2,0.3$, or 0.4 , respectively. At $\hat{p}=0.1$, only 20 , 9 , or 5 plants would need to be evaluated to attain the same level of precision. In general, larger sample sizes were needed to estimate incidence with the same level of precision in the presence of aggregation than under the assumption of a random distribution of disease (13). However, because $b>1$ in the power law, the power law line falls below the binomial line and fewer samples were required when $\hat{p}<$ 0.003 (Fig. 4B), the point at which the binary power law crossed the line of a theoretical binomial distribution (Fig. 3).

The probability of not observing disease in a series of $N$ observations increased with an increase in heterogeneity for a given level of $p$, as predicted by equations 8 and 9 (Fig. 5A). The probability of not observing disease was 0.003 at $p=0.006$ (10 diseased cones when $n=25$ and $N=60$ ) and $\theta=0.05$, but the probability of not detecting disease was 0.0001 when $p=$ 0.006 for a binomial distribution. In other words, only in 1 of 10,000 transects would disease not be observed if the actual $p$ was equal to 0.006 when $N=60$ and $n=25$ under a binomial distribution (Fig. 5A). When $\theta=0.05$ or $\theta=0.02$, the probability of not detecting disease was 0.0001 when $p=0.093$ or 0.0076 (equations 10 and 11 ; Fig. 5B).

\section{DISCUSSION}

Data from 6 years of intensive surveys collected from 41 commercial hop yards in Oregon and Washington were used to characterize the spatial pattern of diseased cones. Point pattern and binary power law analyses indicated a low degree of aggregation of disease within plants, with the extent of aggregation being a function of disease incidence. Correlation-based analyses indicated a largely random pattern of disease among neighboring plants. Spatial correlations did exist in 11 to $20 \%$ of data sets, but were unrelated with any of the specific variables measured.

Regional differences in climate, inoculum density, and grower cultural practices are known to contribute to differences in incidence of hop powdery mildew between Oregon and Washington (32). Field surveys conducted from 1999 to 2002 found that flagshoots (overwintering buds or shoots that emerge completely colonized by the pathogen) were produced on approximately $1.8 \%$ of the plants in Washington but only $0.02 \%$ of the plants in Oregon (18), and that the incidence of powdery mildew on leaves was greater in Washington compared with yards in Oregon $(31,32)$. Leaf infections (initiated by flagshoots) provide the inoculum for cone infections $(20,24,32)$; therefore, the greater prevalence of flagshoots and higher incidence of disease on leaves in Washington likely contributed to differences in incidence of diseased cones between these regions. However, regional differences in climate and inoculum density do not explain why the incidence of diseased cones has increased over time in Washington but not in Oregon. One possible explanation for this increase is that inoculum density is increasing in Washington because of enhanced overwintering of the pathogen. However, surveys conducted from 2003 to 2005 have found that the prevalence of flagshoots in Washington has remained near or below the incidence detected from 1999 to 2002 (W. F. Mahaffee and D. H. Gent, unpublished data). A more likely reason for the increase in disease is that grower tolerance of diseased cones of bittering hops intended for the extract market is increasing. The Washington hops yards included in this study were planted mainly to cultivars used in the extract market, whereas hop yards in Oregon were planted solely with aroma cultivars. Growers producing hops for the $\alpha$-acid extract market have reduced disease management inputs due to a decreased value of the crop, and cone appearance is of secondary impor- tance to $\alpha$-acid content and yield. Mahaffee et al. (18) noted that some growers in Washington discontinue fungicide applications in mid-July, nearly 2 months before harvest. Growers of aroma cultivars, however, must manage the disease more stringently to maintain relatively disease-free cones because aroma hops are evaluated on appearance and quality $(18,20,24)$. For instance, a late-season epidemic of hop powdery mildew on the aroma cv. Willamette in Oregon in 2001 caused nearly $50 \%$ of the crop to be rejected by the contracting brewery (18). However, similar damage from powdery mildew is generally acceptable in Washington on cultivars intended for the $\alpha$-acid extract market.

Turechek and Mahaffee (31) found that the pattern of the incidence of powdery mildew within and among plants is largely random, and speculated that random patterns of disease incidence may be a common attribute of powdery mildew diseases in perennial systems, such as apple (Malus pumila) (33). In the current study, the incidence of powdery mildew on cones within

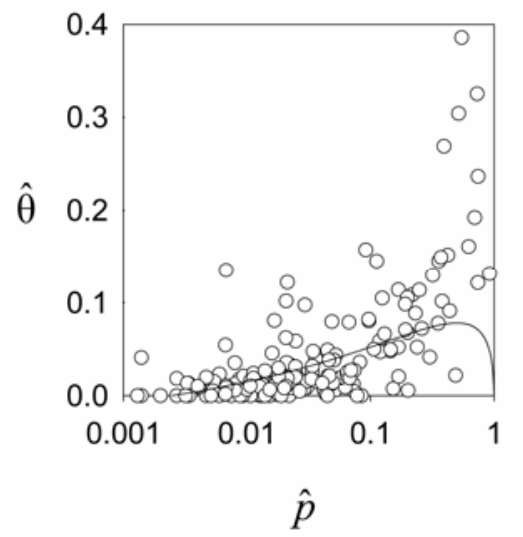

Fig. 2. Relationship between disease incidence $(\hat{p})$ and the parameter $\hat{\theta}$ of the beta-binomial distribution for the incidence of hop powdery mildew on cones in 221 transects sampled from 41 commercial hop yards in Oregon and Washington from 2000 to 2005 (excluding data from 20 transects in which the incidence of disease was 0 ). The solid line is the predicted relationship, which was derived from equation 6 shown in the main text, using least squares estimates of $A_{p}$ and $b$ of the binary power law (equation 4 ) pooled over all years $(11,28)$

Table 3. Mean value and standard error of the beta-binomial parameter $\hat{\theta}$ for incidence of hop cones with powdery mildew sampled from commercial hop yards in Oregon and Washington (Lower Valley, Moxee, and Yakima Indian Reservation [YIR]) ${ }^{\mathrm{a}}$

\begin{tabular}{|c|c|c|c|c|c|c|c|c|c|c|}
\hline \multirow[b]{2}{*}{ Year ${ }^{b}$} & \multicolumn{2}{|c|}{ Oregon } & \multicolumn{2}{|c|}{ Lower Valley } & \multicolumn{2}{|c|}{ Moxee } & \multicolumn{2}{|c|}{ YIR } & \multicolumn{2}{|c|}{ Total } \\
\hline & $\hat{\theta}$ & $\mathbf{T}$ & $\hat{\theta}$ & $\mathbf{T}$ & $\hat{\theta}$ & $\mathbf{T}$ & $\hat{\theta}$ & $\mathbf{T}$ & $\hat{\theta}$ & $\mathbf{T}$ \\
\hline 2000 & $0.02(0.003)$ & 45 & $0.02(0.004)$ & 32 & $0.04(0.014)$ & 15 & $0.03(0.008)$ & 20 & $0.02(0.003)$ & 112 \\
\hline 2001 & $0.01(0.005)$ & 13 & $0.02(0.009)$ & 9 & $0.03(0.017)$ & 6 & $0.02(0.005)$ & 11 & $0.02(0.004)$ & 39 \\
\hline 2002 & $0.03(0.035)$ & 14 & $0.06(0.258)$ & 2 & $0.13(\ldots)$ & 1 & $0.15(0.054)$ & 4 & $0.08(0.02)$ & 21 \\
\hline 2003-04 & $0.04(0.014)$ & 9 & $0.08(0.042)$ & 2 & $0.08(0.047)$ & 4 & $0.08(0.029)$ & 3 & $0.06(0.014)$ & 18 \\
\hline 2005 & $0.01(0.058)$ & 4 & $0.18(0.071)$ & 3 & $0.20(0.040)$ & 2 & $0.19(0.041)$ & 3 & $0.13(0.032)$ & 12 \\
\hline Total & $0.02(0.003)$ & 85 & $0.04(0.075)$ & 48 & $0.06(0.013)$ & 28 & $0.05(0.011)$ & 41 & $0.04(0.003)$ & 202 \\
\hline
\end{tabular}

${ }^{a}$ The heterogeneity parameter of the beta-binomial distribution $(\hat{\theta})$ is an index of aggregation that provides a measure of variation in disease incidence among sampling units (11), and $\mathrm{T}=$ number of data sets (transects sampled through hop yards).

${ }^{\mathrm{b}}$ Field sites in each region were combined in 2003 and 2004 because of the small number of fields assessed in these years. 

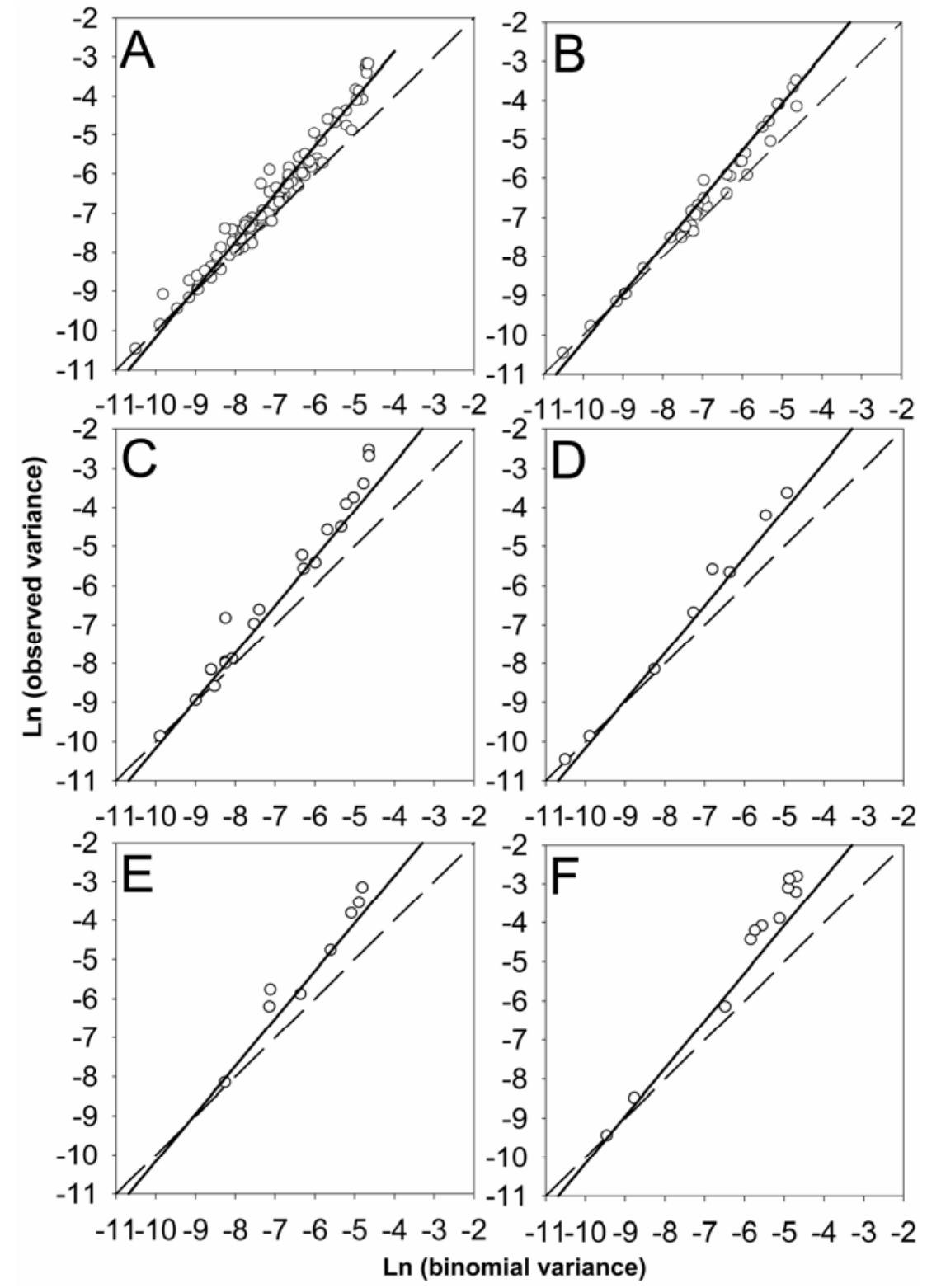

Fig. 3. A-F, Relationship between the logarithm of the observed variance and the logarithm of the theoretical variance for a binomial distribution of the incidence of hop cones with powdery mildew in Oregon and Washington hop yards sampled from 2000 to 2005. The solid line is the least squares regression fit to the data, and the dashed line is the theoretical binomial distribution with power law parameters of $b=1$ and $\ln \left(A_{p}\right)=0$ (11). Data were obtained from 221 transects collected in 41 commercial hop yards, excluding data from 20 transects in which the incidence of disease was 0 .

Table 4. Slope and intercept parameter estimates of the binary power law fit to the incidence of hop cones with powdery mildew sampled from commercial hop yards in Oregon and Washington

\begin{tabular}{lrccc}
\hline Year & $\mathbf{d f}^{\mathbf{a}}$ & $\hat{b}(\mathbf{S E})^{\mathbf{b}}$ & $\ln \left(\hat{A}_{p}\right)(\mathbf{S E})^{\mathbf{b}}$ & $\boldsymbol{R}^{\mathbf{2}}$ \\
\hline 2000 & 111 & $1.16(0.019)$ & $1.50(0.143)$ & 97.1 \\
2001 & 38 & $1.24(0.088)$ & $2.14(0.636)$ & 98.2 \\
2002 & 20 & $1.30(0.049)$ & $2.83(0.351)$ & 97.4 \\
2003 & 8 & $1.25(0.040)$ & $2.47(0.316)$ & 99.3 \\
2004 & 7 & $1.28(0.117)$ & $2.68(0.732)$ & 95.2 \\
2005 & 11 & $1.37(0.049)$ & $3.37(0.318)$ & 98.7 \\
Pooled & 199 & $1.22(0.016)$ & $2.01(0.117)$ & 96.7 \\
\hline
\end{tabular}

${ }^{a}$ Degrees of freedom (df) for regression.

b $\hat{b}$ and $\ln \left(\hat{A}_{p}\right)$ are slope and intercept estimates, respectively, calculated as per (10). SE = standard error of the mean. Data from 2003 and 2004 are shown separately because data sets from all regions were used in the regression analysis and sufficient data was available to estimate parameters of the binary power law. $P$ value for the effect of hop cultivar or geographic region of the hop yard on the intercept $\ln \left(\hat{A}_{p}\right)$ and slope $\hat{b}$ of equation 5 shown in the text and calculated as per Turechek and Madden (29). plants was slightly more aggregated than the incidence of the disease on leaves (31), as evidenced by greater values of $\theta, D$, and parameter estimates of the binary power law. However, runs analyses and spatial autocorrelation statistics still indicated a largely random pattern of disease beyond the scale of individual sampling units (plants). The increase in disease heterogeneity observed on cones may be attributed to cone tissue being susceptible for a longer period of time relative to leaf tissue, and the proximity of cones to existing inoculum (i.e., sporulating leaf lesions). Young, unfurled hop leaves are most susceptible to powdery mildew, but their susceptibility decreases with time and leaves generally are not susceptible to infection 15 days after they emerge $(24,32)$. Such ontogenic resistance is absent in burrs and cones (25). Leaves also become increasingly resistant to infection with extended exposure (6 h or more) to temperatures of $30^{\circ} \mathrm{C}$ or higher (19). It is possible that during periods of extended high temperatures-common in the hop-production regions of Oregon and Washington during the summer-hop leaves emerge and escape infection because of unfavorable weather for the pathogen and disease. Hop cones, however, develop later in the season when temperatures generally are more favorable for disease, remain susceptible to infection until harvest $(20,24,25)$, and often are produced in close proximity to infected leaves. Cones located on plants with a high disease intensity on leaves have a greater probability of becoming infected than cones on plants with lower disease intensity. Additionally, the number of cones infected will increase more rapidly on these plants as predicted under logistic growth, a reasonable assumption for a polycyclic disease (2). In this study, aggregation is characterized simply by differences in the number of infected cones among plants based on point-pattern analyses; therefore, the longer window of susceptibility of cones versus leaves, paired with the proximity of cones to potential sources of inoculum, appears to result in conditions that lead to a greater degree of spatial aggregation for infected cones than infected leaves.

The information generated in this study on spatial heterogeneity of diseased cones was used to create sampling curves for fixed sample sizes $(5,6)$. The sample size $(N=60)$ utilized in this study should estimate disease at $p=0.01$ with precision $C=$ 0.3 , but is insufficient to estimate $p$ below this incidence at this level of precision. However, a limitation of this approach is the necessity for a reasonable estimate of $p$ in order to use these curves. In other words, an initial estimate must be provided of the very value that is being estimated. Providing too high an estimate will lead to an imprecise estimate, and a low estimate will lead to oversampling at the specified 
level of precision. A solution is to derive an estimate of $p$ based on the incidence for powdery mildew on leaves. The relationship between diseased cones and leaves, as shown in Figure 5 of Turechek et al. (32), could be used as an approximate initial estimate. An alternative solution is to develop sequential sampling curves where no knowledge of disease incidence is needed before sampling commences.

The probability of observing no diseased cones in a sample of size $\mathrm{Nn}$ was derived with equations 8 and 9 , and is useful for estimating when the tolerance for disease is zero or close to zero. This is analogous to inverse sampling sometimes used in seed sampling protocols or quarantine procedures (15). Because of the low degree of aggregation of disease within plants, a slightly larger sample size is required to calculate this probability than if disease were distributed randomly. According to the power law results, heterogeneity varies with $\hat{p}$, which may be problematic in practice because equations 8 and 10 assume constant $\theta$. Consequently, the calculations for $p, P$, and $N$ will be approximate depending on the true $\theta$. It is possible to substitute equation 6 for $\theta$ in equations 8 and 10; however, with this substitution it is very difficult to rearrange these equations in terms of $p$ for an upper confidence limit because equation 6 is a complicated function of $p$. Nonetheless, these relationships may have practical applications for hop buyers who may reject some lots of cones if the probability of disease exceeds an established level, or pay a premium for disease-free crops.

The patterns of disease assessed in this study were for incidence of diseased cones within and between plants. Cones assessed from an individual plant were obtained from a bulked sample of cones collected at multiple heights, although the incidence of hop powdery mildew may not be spatially uniform at all heights in the canopy. The pattern of diseased cones in threedimensional space was beyond the scope of this study, but may affect disease heterogeneity. Knowledge of the spatial patterns of disease among cones at different heights on a plant may provide insights into disease epidemiology and management, and will be investigated in future research.

Another important finding of this research was the apparent absence of cleistothecia of $P$. macularis on cones in the Pacific Northwest. In the first report of hop powdery mildew in the Pacific Northwest, Ocamb et al. (21) noted that cleistothecia had not been observed in the field. Six years later, this statement still appears to be true. Royal (24) noted that, in England, "Cleistothecia form during virtually every outbreak of the disease and can develop so
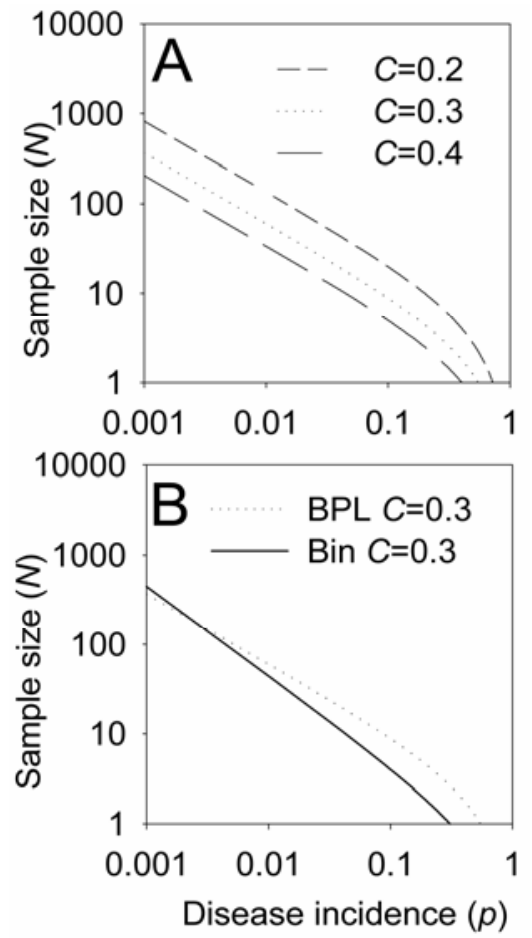

Fig. 4. Sample size required to estimate the incidence $(\hat{p})$ of hop cones with powdery mildew based on the binary power law, with slope ln $\left(\hat{A}_{p}\right)=2.01$ and intercept $\hat{b}=1.22$ (see equation 7 in the text). A, Sampling curves for three levels of precision $(C)$. B, Sampling curves for binomial and binary power law cases at $C=0.3$. The solid line represents the sampling curve for a binomial (random) distribution of disease (6). profusely as to turn infected cones black." More than 300,000 cones were evaluated in this study, and the sexual stage of $P$. macularis was not observed, suggesting that only one mating type of the fungus has been introduced or is prevalent in the Pacific Northwest. Sexual recombination of
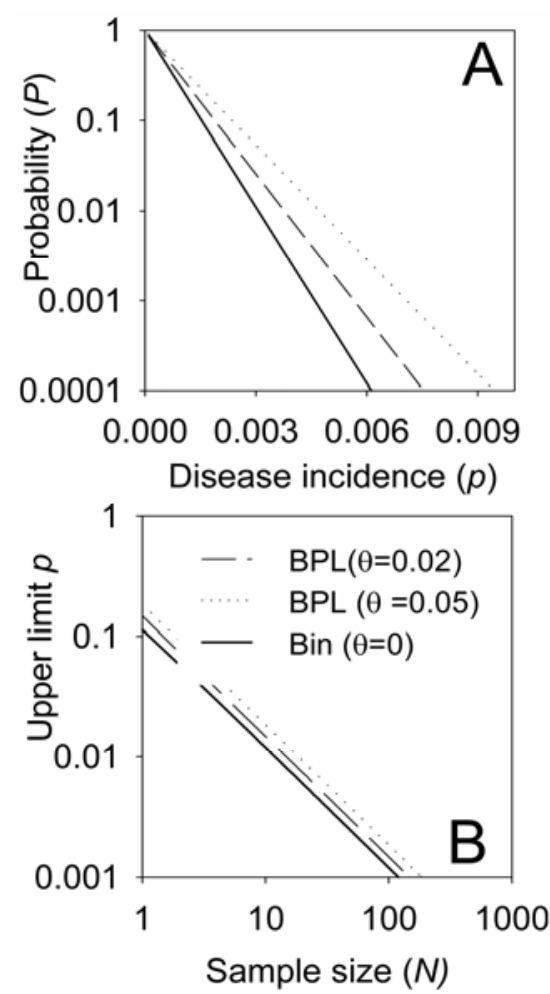

Fig. 5. Sampling curves when the observed incidence of hop cones with powdery mildew is near zero. A, Relationship of disease incidence and the probability of not observing disease among any plants when $N$ (sampling units) $=60$, $n$ (individuals per sampling unit) $=25$, and the heterogeneity parameter of the beta-binomial distribution, $\hat{\theta},=0.02$ (Oregon hop yards), 0.05 (Washington hop yards), or 0 (binomial distribution) according to equations 8 and 9 shown in the text. B, Upper limit of a $95 \%$ confidence interval for the incidence of hop cones with powdery mildew $(p)$ in relation to sample size $(N)$, when $n=25$ and $\hat{\theta}=0.02$ (Oregon), 0.05 (Washington), or 0 (binomial distribution) according to equations 10 and 11 in the text (15).

Table 5. Deviance and significance level of hop cultivar, geographic region of the yard, and year of sampling on the intercept $\left(\ln \left[\hat{A}_{p}\right]\right)$ and slope $(\hat{b})$ parameters of the binary power law for the incidence of hop powdery mildew on cones sampled from commercial hop yards in Oregon and Washington in 2000 to 2005 , as determined by analysis of covariance ${ }^{\mathrm{a}}$

\begin{tabular}{|c|c|c|c|c|c|c|c|c|c|c|}
\hline \multirow[b]{2}{*}{ Factor } & \multicolumn{2}{|c|}{$d f^{b}$} & \multicolumn{4}{|c|}{$\ln \left(\hat{A}_{p}\right)$} & \multicolumn{4}{|c|}{$\hat{b}$} \\
\hline & Deviance & Factor & Dev. $^{c}$ & Diff. $^{d}$ & $F^{\mathrm{e}}$ & $P$ & Dev. & Diff. & $F$ & $P$ \\
\hline Power law & 199 & $\ldots$ & 25.176 & & & & 25.176 & & & \\
\hline Cultivar & 198 & 1 & 25.150 & 0.026 & 0.205 & 0.651 & 25.043 & 0.133 & 1.052 & 0.306 \\
\hline Region & 196 & 3 & 25.024 & 0.152 & 0.397 & 0.755 & 24.922 & 0.254 & 0.666 & 0.574 \\
\hline Year & 194 & 5 & 18.936 & 6.24 & 12.786 & 0.000 & 20.832 & 4.340 & 8.083 & 0.000 \\
\hline
\end{tabular}

a Intercept and slope parameters of the binary power law were estimated by least square regression analysis of data fit to equation 5 .

${ }^{b}$ Degrees of freedom (df) for the analyses of covariance.

c Dev. = deviance for the covariance model.

${ }^{\mathrm{d}}$ Diff. $=$ difference between the deviance of the binary power law model versus the binary power law model with each factor included in the analyses first as an intercept and then as a slope.

e Significance level for the difference between deviances of the binary power law model versus binary power law model with each factor as determined by an $F$ test, where $F=$ (factor dev./df factor)/(model dev./df deviance) (29). 
the pathogen may have important implications for management of hop powdery mildew and will be monitored in future research. The sampling strategies developed in this research should facilitate these efforts.

\section{ACKNOWLEDGMENTS}

Financial support was provided by USDA-ARS CRIS 303-5358-22000-030-00D and 5358-21000035-00) and grants from the Hop Research Council, Washington State Commission on Pesticide, and the Washington Hop Commission. We thank L. du Toit and H. Schwartz for their critical review of an earlier draft of the manuscript, L. Madden for helpful suggestions on the binary power law, the many individuals that assisted in disease assessments, and the hop growers that allowed us access to their yards and provided numerous insights.

\section{LITERATURE CITED}

1. Barth, H. J., Klinke, C., and Schmidt, C. 1994. The Hop Atlas. Joh. Barth and Sohn, Nuremberg, Germany.

2. Campbell, C. L., and Madden, L. V. 1990. Introduction to Plant Disease Epidemiology. John Wiley and Sons, New York.

3. Hughes, G. 1988. Spatial heterogeneity in crop loss assessment models. Phytopathology 78:883-884.

4. Hughes, G. 1999. Sampling for decision making in crop loss assessment and pest management: introduction. Phytopathology 89:10801083.

5. Hughes, G., and Madden, L. V. 1992. Aggregation and incidence of disease. Plant Pathol. 41:657-60.

6. Hughes, G., and Madden, L. V. 1994. Aggregation and incidence of disease: Some implications for sampling. Asp. Appl. Biol. 37:25-31.

7. Hughes, G., Madden, L. V., and Munkvold, G. P. 1996. Cluster sampling for disease incidence data. Phytopathology 86:132-137.

8. Hughes, G., McRoberts, N., Madden, L. V., and Gottwald, T. R. 1997. Relationships between disease incidence at two levels in a spatial hierarchy. Phytopathology 87:542-550.

9. MacKinnon, D. 2006. USA Hops: 2005 Statis- tical Report. Hop Growers of America, Yakima, WA

10. Madden, L. V., and Hughes, G. 1994. BBDcomputer software for fitting the beta-binomial distribution to disease incidence data. Plant Dis. 78:536-540.

11. Madden, L. V., and Hughes, G. 1995. Plant disease incidence: distribution, heterogeneity, and temporal analysis. Annu. Rev. Phytopathol. 33:529-564.

12. Madden, L. V., and Hughes, G. 1999. An effective sample size for predicting plant disease incidence in a spatial hierarchy. Phytopathology 89:770-781.

13. Madden, L. V., and Hughes, G. 1999. Sampling for plant disease incidence. Phytopathology 89:1088-1113.

14. Madden, L. V., Hughes, G., and Ellis, M. A. 1995. Spatial heterogeneity of the incidence of grape downy mildew. Phytopathology 85:269275.

15. Madden, L. V., Hughes, G., and Munkvold, G. P. 1996. Plant disease incidence: Inverse sampling, sequential sampling, and confidence intervals when observed mean incidence is zero. Crop Prot. 15:621-632.

16. Madden, L. V., Louie, R., Abt, J. J., and Knoke, J. K. 1982. Evaluation of tests for randomness of infected plants. Phytopathology 72:195-198.

17. Madden, L. V., Turechek, W. W., and Nita, M. 2002. Evaluation of generalized linear mixed models for analyzing disease incidence data obtained in designed experiments. Plant Dis. 86:316-325.

18. Mahaffee, W. F., Thomas, C. S., Turechek, W. W., Ocamb, C. M., Nelson, M. E., Fox, A., and Gubler, W. D. 2003. Responding to an introduced pathogen: Podosphaera macularis (hop powdery mildew) in the Pacific Northwest. Online. Plant Health Progress doi:10.1094/ PHP-2003-1113-07-RV.

19. Mahaffee, W. F., Turechek, W. W., and Ocamb, C. M. 2003. Effect of variable temperature on infection severity of Podosphaera macularis on hops. Phytopathology 93:1587-1592.

20. Neve, R. A. 1991. Hops. Chapman and Hall, London.

21. Ocamb, C., Klein, R., Barbour, J., Griesbach, J., and Mahaffee, W. 1999. First report of hop powdery mildew in the Pacific Northwest. Plant Dis. 83:1072.

22. Paul, S. R., Liang, K. Y., and Self, S. G. 1989. On testing departure from binomial and multinomial assumptions. Biometrics 45:231-236.

23. Pethybridge, S. J., Esker, P., Hay, F., Wilson, C., and Nutter, F. W. 2005. Spatiotemporal description of epidemics caused by Phoma ligulicola in Tasmanian pyrethrum fields. Phytopathology 95:648-658.

24. Royal, D. J. 1978. Powdery mildew of the hop. Pages 381-409 in: The Powdery Mildews. D M. Spencer, ed. Academic Press, London.

25. Seigner, E., Seefelder, S., Haugg, B., Engelhard, B., Hasyn, S., and Felsenstein, F. G 2003. Potential of powdery mildew (Sphaerotheca humuli) to infect hops (Humulus lupu$l u s$ ) in various developmental stages. Gesunde Pflanz. 55:29-33.

26. Taylor, L. R. 1961. Aggregation, variance, and the mean. Nature 189:732-735.

27. Taylor, L. R., Woiwod, I. P., and Perry, J. N. 1978. The density-dependence of spatial behaviour and the rarity of randomness. J. Anim Ecol. 47:383-406

28. Turechek, W. W., Ellis, M. A., and Madden, L. V. 2001. Sequential sampling for incidence of Phomopsis leaf blight of strawberry. Phytopathology 91:336-347.

29. Turechek, W. W., and Madden, L. V. 1999. Spatial pattern analysis of strawberry leaf blight in perennial production systems. Phytopathology 89:421-433.

30. Turechek, W. W., and Madden, L. V. 1999. Spatial pattern analysis and sequential sampling for the incidence of leaf spot on strawberry in Ohio. Plant Dis. 83:992-1000.

31. Turechek, W. W., and Mahaffee, W. F. 2004 Spatial pattern analysis of hop powdery mildew in the Pacific Northwest: Implications for sampling. Phytopathology 94:1116-1128.

32. Turechek, W. W., Mahaffee, W. F., and Ocamb, C. M. 2001. Development of management strategies for hop powdery mildew in the $\mathrm{Pa}$ cific Northwest. Online. Plant Health Progress doi:10.1094/PHP-2001-0313-01-RS

33. Xu, X. M., and Madden, L. V. 2002. Incidence and density relationships of powdery mildew on apple. Phytopathology 92:1005-1014. 